\title{
Sectionally Pseudocomplemented Residual LATTICE
}

\author{
Md. Zaidur Rahman ${ }^{1}$, Md. Abul Kalam Azad ${ }^{1}$ and Md. Nazmul Hasan. ${ }^{2}$ \\ Dept. of Mathematics ${ }^{1}$ \\ Khulna University of Engineering and Technology \\ Dept. of Mathematics ${ }^{2}$ \\ Moheshpur Govt. College, Moheshpur, Jhenaidah. \\ E-mail mzrahman1968@gmail.com, azadmath.azad8@gmail.com
}

\begin{abstract}
At first, we recall the basic concept, By a residual lattice is meant an algebra $L=(L, \vee, \wedge, *, \circ, 0,1)$ such that

(i) $L=(L, \vee, \wedge, 0,1)$ is a bounded lattice,

(ii) $L=(L, *, 1)$ is a commutative monoid,

(iii) it satisfies the so-called adjoin ness property: $(x \vee y) * z=y$ if and only if $y \leq z \leq x \circ y$

Let us note [7] that $x \vee y$ is the greatest element of the set $(x \vee y) * z=y$

Moreover, if we consider $x * y=x \wedge y$, then $x \circ y$ is the relative pseudo-complement of $x$ with respect to $y$, i. e., for $*=\wedge$ residuated lattices are just relatively pseudo-complemented lattices. The identities characterizing sectionally pseudocomplemented lattices are presented in [3] i.e. the class of these lattices is a variety in the signature $\{\vee, \wedge, \circ, 1\}$. We are going to apply a similar approach for the adjointness property:
\end{abstract}

Key words: Residuated lattice, non Distributive, Residuated Abeliean, commutative monoid:

\section{Introduction}

Residuated lattices were introduced by Ward and Dilworth [5] and studied by several authors. Two monographs contain a compendium on residuated lattices. They are that by Blyth and Janowitz [1] (where it is renamed as a residuated Abelian semi-group with a unit) and the book by R. Belohavek [7]. In this short note we will compare a certain modification of a residuated lattice with already introduced [2], [3]. At first, we recall the basic concept:

Definition 1. A lattice $L=(L, \vee, \wedge, 1)$ with the greatest element 1 is sectionally pseudocomplemented if each interval $[y, 1]$ is a pseudo-complemented lattice.

Date of submission : 21.08.2010

Date of acceptance : 07.04.2011
From now on, denote by $x \vee y$ the pseudocomplement of $x \vee y$ in the interval [y, 1].

Naturally, $x \vee y \in[y, 1]$ thus $L=(L ; \vee, \wedge, 1)$ is sectionally pseudo-complemented if and only if "o" is an (everywhere defined) operation on L.

Definition 2. An algebra $L=(L ; \vee, \wedge, *, \circ, 1)$ is called a sectionally residuated lattice if

(i) $L=(L, \vee, \wedge, 0,1)$ is a lattice with the greatest element 1 ;

(ii) $L=(L, *, 1)$ is a commutative monoid ;

(iii) it satisfies the sectional adjointness property: $(x \vee y) * z=y$ if and only if $y \leq z \leq x \circ y$

Lemma 1.1 Let $L=(L ; \vee, \wedge, *, \circ, 1)$ be a sectionally residuated lattice. Then $x * y$ is the greatest element of the set $\{z ;(x \vee y) * z=y\}$

This immediately yields the following facts:

$(x \vee y) *(x \circ y)=y$,

$(x \vee y) * y=y$,

$y \leq x \circ y$,

Lemma 1.2 Let $L=(L ; \vee, \wedge, *, \circ, 1) \quad$ be a sectionally residuated lattice. Then $x \leq y$, if and only If $x \circ y=1$

Proof: Suppose $x \leq y$, Then $x \vee y=y$, and by Lemma 1.1, $x \circ y$ is the greatest element of the set $\{z ; y * z=y\} \quad$ By Definition 2, $y * 1=1$ thus $x \circ y=1$. Conversely,

Suppose $x \circ y=1$.Then, by [1], we have $y=(x \vee y) *(x \circ y)=(x \vee y) * 1=x \vee y$ 
whence $x \leq y$

Lemma 1.3 In a sectionally residuated lattice, the following identities are satisfied:

and $1 \circ \mathrm{x}=\mathrm{x}$

Proof: The first three identities follow directly by Lemma 1.2. Further, by Lemma 1.1,

$1 \circ x$ is the greatest element of the set $\{z ; 1 * z=x\}=\{x\}$ thus $1 \circ x=x$

Lemma 1.4 In a sectionally residuated lattice, $a * b=a$ if and only if $a=b$

Proof: Putting $x=y=a$ and $z=b$ in the sectional adjointness property, the assumption $a * b=a$ yields $(a \vee a) * b$ iff $a \leq b \leq a \circ a=1$ thus $a \leq b$

Conversely, $a \leq b$ implies by Lemma 3 $a \leq b \leq 1=a \circ a$ and, by sectional adjointness, $a * b=(a \vee a) * b=a$

Applying Lemma 1.2 and Lemma 1.4, we get Corollary 1.5 In a sectionally residuated lattice,

(a) $x * y=x$ if and only if $x \circ y=1$;

(b) $x * x=x$

Lemma 1.6 In a sectionally residuated lattice, $x \wedge y \leq x * y$.

Proof: By [3] we have $x \wedge y \leq x \circ(x \wedge y)$. Applying sectional adjointness, we infer $x *(x \wedge y)=(x \vee(x \wedge y)) *(x \wedge y) \quad$ and, analogously, $y *(x \wedge y)=x \wedge y$. Hence, by Corollary 1.5 (b),

$x * y *(x \wedge y)=x *(x \wedge y) * y *(x \wedge y)$

$=(x \wedge y) *(x \wedge y)=x \wedge x$

and by Lemma 1.4, $x \wedge y \leq x * y$.

Theorem 1.7 Let $L=(L ; \vee, \wedge, *, \circ, 1)$ be a sectionally residuated lattice. Then it is a sectionally pseudo-complemented lattice.
Proof: Replacing y by $x \wedge y$ in the sectional adjointness property, we obtain $x * z=x \wedge y$ iff $x \wedge y \leq z \leq x \circ(x \wedge y)$.

However, $x \circ(x \wedge y)$ is the greatest element of the set $\{t ;(x \vee(x \wedge y)) * t=x \wedge y\}=\{t ; x * t=x \wedge y\}$.

By Lemma 1.4, $x \wedge t \leq x * t=x \wedge y$, thus the greatest $\mathrm{t}$ of this property satisfies $t \geq y$.

Thus $\quad y \leq x \circ(x \wedge y)$, i.e., $x \wedge y \leq y \leq x \circ(x \wedge y)$ and by the sectional adjointness, $x * y=(x \wedge(x \vee y)) * y=x \wedge y$.

Hence, $x \circ y$ is the pseudo-complement of $x \vee y$ in the interval $[y, 1]$

\section{Conclusion}

It is well known that every relatively pseudocomplemented lattice is distributive.

An extension of relative pseudocomplementation for the non-distributive case was already involved in [3], [4]:

\section{Reffrences}

[1] Blyth, T.S. and Janowttz, M.F. : Residuation Theory, Pergamon Press, Oxford, 1972.

[2] Chajda, I. and Halas, R: Sectionally pseudocomplemented lattice and semilattice, Advances in algebra, Springer Verlag, 2003, 282290.

[3] Chajda, I.: An extension of relative pseudocomplemention to non distributive lattices, Acta Sci. Math(Szeged), 69 (2003), 491-496.

[4] Chajda, I. and Radeleczki, S.: On varities defined by pseudo-complemented nondistributive lattices, Publ. Math. (Debrecen). 63 (2003), 737-750.

[5] Ward, M. and Dilworth, R.P.: Residuated lattices, Trans.Amer.Math. Soc., 45(1939), 335-354.

[6] Chajda, I. : Sectionally Residuated lattices, Miskolc Mathmatical notes, Vol. 6 (2005), no. 1,pp. 27-30

[7] R. Belohavek; Fuzzy relational systems, Kluwer Academic/ Plemun Publ., New York, Boston, Dordrecht, London, Moscow, 2002 